\title{
Conhecimentos e práticas em saúde bucal com crianças hospitalizadas com câncer
}

\author{
Knowledge and practices of oral health \\ on hospitalized children with cancer
}

\author{
Aline $\mathrm{M}$ ay Barbosa ${ }^{1}$ \\ Dayane M achado Ribeiro ${ }^{2}$ \\ Angela Scarparo Caldo-Teixeira ${ }^{3}$
}

${ }^{1}$ Curso de O dontologia, Universidade do Sul de Santa Catarina. Av. José Acácio M oreira 787, Bairro Dehon. 88704-900 Tubarão SC. yamenila@yahoo.com.br ${ }^{2}$ Departamento de

Estomatologia,

UniversidadeFederal de

Santa Catarina.

${ }^{3}$ Departamento deFormação

Específica, Faculdadede

Odontologia do Pólo

Universitário de Nova

Friburgo, U niversidade

Federal Fluminense.
Abstract The aim of this study was to evaluate the knowledge and practices of oral health $(\mathrm{OH})$ in hospitalized children with cancer. The sample was composed by the nurse team, caretakers and children. It was applied a questionnaire concerning the general knowledge about $\mathrm{OH}$, methods and instruments used for oral hygiene $(\mathrm{OH})$. According to the results, the responsible of $\mathrm{OH}$ of children are the caretakers $(90.7 \%)$ who receive instructionsfrom thenurseteam in $21.4 \%$ of cases. As for the oral cavity discomfort, the nurse team reported that all patients exhibited clinical manifestations while the caretakers reported a different number, $62.8 \%$ of cases. All participants considered important having a dentist in the oncology sector. According to the results obtained, it was possible to conclude that there is no oral health protocol for hospitalized children with cancer and that the most frequent oral manifestations among patients going through antineoplastic treatment were: mucositis, nausea, vomit, xerostomy and lack of sense of taste.

Key words Dentistry hospital, O ncology, H ealth education
Resumo 0 objetivo do estudo foi avaliar os conhecimentos e práticas em saúde bucal (SB) com crianças hospitalizadas com câncer. A amostra foi composta pela equipe de enfermagem, cuidadores e crianças. Foi aplicado um questionário relacionado ao conhecimento geral sobre SB, métodos e instrumentos utilizados para a higiene oral $(\mathrm{HO})$ e dados socioeconômicos. Baseado nos resultados, quem realiza a $\mathrm{HO}$ das crianças são os cuidadores $(90,7 \%)$, que receberam orientações da equipe de enfermagem em $21,4 \%$ dos casos. Com relação ao desconforto na cavidade bucal, a equipe de enfermagem reportou que todos apresentaram manifestaçõesclínicas, enquanto apenas $62,8 \%$ doscuidadores reportaram casos. Todos os participantes consideram importante haver um CD no setor de oncol ogia. Pôde- seconcluir quenão existeum protocolo de cuidados com a higiene bucal de crianças hospitalizadas com câncer eque as manifestações bucais mais frequentes entre os pacientes em tratamento antineoplásico foram: mucosite, enjoos, vômitos, xerostomia e ausência de paladar. Palavras-chave Odontologia hospitalar, Oncologia, Educação em saúde 
Introdução

As práticas dehigienebucal desempenham importante papel na prevenção de doenças bucais ${ }^{1-3}$. Há, atualmente, uma grande variedade de métodos disponíveis para a remoção mecânica da placa bacteriana. A escovação dental manual permane ce, no entanto, sendo o método de eleição para se alcançar uma boa higiene bucal ${ }^{4}$.

Deacordo com H onkala ${ }^{5}$, é recomendado escovar os dentes duas vezes ao dia, sendo considerados "escovadores regulares" todos aqueles que escovarem os dentes ao menos uma vez ao dia.

As doenças bucais constituem atual menteum importante problema de saúde pública, não somente devido à sua alta prevalência, mas também pelo seu impacto em nível individual ecole tivo, em termos de dor, desconforto, limitações sociais e funcionais, o que afeta a qualidade de vida do indivíduo ${ }^{6}$.

A doença cárie juntamente com as doenças periodontais constituem as afecções de maior prevalência na cavidade bucal, e também podem acometer crianças e levar à perda precoce de elementos dentais quando não tratadas adequadamente?

De acordo com Lascala $a^{8}$, a constante evolução dos conceitos de promoção de saúde e do entendimento epidemiológico demultifatoriedade e a proposta de tratamento segundo o risco à cárie fizeram com que a prática odontológica se voltasse para a promoção de saúde, enfatizando a necessidade de atuação nos agentes predisponentes ou causadores das doenças e não somente no tratamento cirúrgico-restaurador.

O cuidado com a saúde bucal de crianças envolve vários setores, tais como: a família, a sociedade, as políticas governamentais e também 0 cirurgião-dentista. Agindo em conjunto, esses setores buscam proporcionar-Ihes saúde?

A odontologia baseada na promoção de saúde a uma população infantil específica, como pacientes acometidos pelo câncer, tem papel fundamental no restabelecimento da saúde geral e, consequentemente, na qualidade de vida dessas crianças.

No Brasil, as estatísticas sobre as neoplasias infantis têm sido pouco analisadas na literatura nacional, apesar da existência de fontes de dados, tais como o Sistema de Informação de M ortalidade do M inistério da Saúde e os Registros de Câncer de Base Populacional em funcionamento no país. Sabe-se que, em 1994, as neoplasias foram responsáveis por $8 \%$ dos óbitos entre as crianças de 1 a 14 anos, representando, assim, a quarta causa de morte (excluindo os óbitos por afecções mal definidas), sucedendo as causas externas, as doenças do aparelho respiratório e as doenças infecciosas ${ }^{10}$.

Dos óbitos por neoplasias na infância, as leucemias representam a maior causa, sendo responsáveis por 39\% das mortes na Europa e por $50 \%$ nas Américas, Oceania e Ásia. 0 declínio observado, em diversos países, nos coeficientes de mortalidade por neoplasias em menores de 15 anos parece ser devido, em grande parte, ao aumento da probabilidade de sobrevida para a maioria dos casos com tumores infantis, ou seja, ao acréscimo na porcentagem de crianças vivas com neoplasias após um determinado período de tempo, em decorrência de diagnósticos mais precoces e maior sucesso nas intervenções terapêuticas (radioterapia, quimioterapia, cirurgia, transplante de medula óssea). Os recentes avanços no uso dos medicamentos quimioterápicose o emprego de esquemas combinados de drogas permitiram elevar a sobrevida de crianças com neoplasias, particularmente as diagnosticadas hematológicas ${ }^{10}$.

Em quase todos os grupos de diagnóstico, tem-se apresentado aumento nas probabilidades de sobrevida. N o entanto, pode ocorrer uma acentuada variabilidade entre as probabilidades de sobrevida dos pacientes diagnosticados com diferentes tumores. Tais variações derivam da história natural da doença, conforme o órgão afetado e das respostas variadas à terapêutica antineoplásica. As neoplasias pediátricas mostram-se habitual mente fatais quando não tratadas adequadamente ou em tempo hábil ${ }^{10}$.

Apesar de, em muitos países, ter-se verificado aumento na incidência das neoplasias em menores de 15 anos e de o prognóstico para vários tumores infantis ainda estar aquém do desejado, sabe-se que a sobrevida das crianças com câncer émelhor hojeem dia. Particularmentepara alguns tipos histológicos, 0 aumento da sobrevida fez-se de forma mais marcante ${ }^{10}$.

Atual mente, $77 \%$ das crianças acometidas por câncer podem ser curadas se diagnosticadas precocementeetratadas em centros especializados ${ }^{11}$.

Um dos principais recursos utilizados no tratamento oncológico infantil é a quimioterapia isolada ou associada à cirurgia e à radioterapia. A função principal das terapias antineoplásicas é a destruição das células malignas, preferencialmente quando estão na fase de mitose. Entretanto, células da mucosa bucal e gastrointestinal, medula e pele também apresentam grau de atividade mitótica semelhante e são especialmente 
propensas a manifestar os efeitos secundários dos agentes antineoplásicos ${ }^{12}$.

Durante o tratamento antineoplásico, as alterações na cavidade bucal al cançam maior gravidade, pois tanto a quimioterapia quanto a radioterapia não diferenciam as células neoplásicas das células normais ${ }^{13}$.

Os principais efeitos colaterais da quimioterapia são a mucosite, a xerostomia temporária e a imunodepressão, possibilitando infecções dentárias ou oportunistas. Observam-se também hemorragias gengivais decorrentes da plaquetopenia e distúrbios na formação dos germes dentários quando a quimioterapia é administrada na fase de odontogênese ${ }^{14-16}$.

O serviço de oncologia pediátrica do $\mathrm{H}$ ospital Infantil Joana de Gusmão (HIJG - Santa Catarina) tem em sua trajetória algumas centenas decrianças tratadas, eapresenta assistência odontológica e acompanhamento clínico de rotina. Porém, não são realizadas a quantificação e qualificação dessas alterações ocasionadas na cavidade bucal pelo tratamento desses pacientes.

Desta forma, o objetivo deste estudo foi avaliar os conhecimentos e práticas em saúde bucal em crianças hospitalizadas no setor de oncologia do Hospital Infantil Joana de Gusmão, em de Florianópolis (SC), bem como quantificar equalificar as complicações bucais provenientes do tratamento antineoplásico.

\section{Métodos}

Após aprovação pelo Comitê de Ética em Pesquisa da Universidade do Sul de Santa Catarina (UNISUL) e do Hospital Infantil Joana de Gusmão (HIJG), o estudo foi iniciado. Os participantes assinaram um termo de consentimento livre e esclarecido (TCLE), segundo a resolução 196/96 do Conselho Nacional de Saúde (CNS).

A população do estudo foi composta pela equipe de enfermagem $(n=19)$ do setor de oncologia do HIJG, crianças hospitalizadas $(n=43)$ e cuidadores (acompanhantes; $n=43$ ) das crianças. Os critérios de seleção foram: crianças hospitalizadas e cuidadores - seleção aleatória entre os pacientes em tratamento quimioterápico, totalizando $n=43$; equipe de enfermagem - seleção aleatória dentre aqueles que trabal havam no se tor deoncologia do HIJG eque concordaram em participar do estudo.
Por meio de entrevista, foram aplicados questionários sobre dados gerais (idade, sexo, estado civil, ocupação profissional, etc.), conhecimentos gerais sobre saúde bucal, métodos e instrumentos utilizados para a higienização bucal. Os dados foram coletados por uma única avaliadora, entre os meses de fevereiro e agosto de 2007. Os resultados obtidos foram analisados pela frequência de respostas (análise descritiva) e pelo teste exato de Fisher $(p<0,05)$, nos casos em que uma mesma pergunta gerou respostas discordantes.

\section{Resultados}

0 perfil da população estudada pode ser observado nas tabelas 1 a 3 .

0 diagnóstico maisfrequente, deacordo com os dados obtidos dentre as 43 crianças avaliadas, foi a leucemia linfoblástica aguda (LLA), em 48\% da amostra, seguido por linfoma de Burkitt (7\%), neuroblastoma (7\%) e osteossarcoma (6\%), como descrito na Tabela 1.

Com relação à equipe de enfermagem (Tabela 3), os profissionais trabalhavam há pelo menos quatro anos no HIJG, por trinta horas semanais, e haviam se formado - em média - há 7,68 anos.

Quando questionada sobre as práticas e conhecimentos em saúde bucal (Tabela 4), apesar de $78,94 \%$ da equipe de enfermagem responder que não havia um protocolo de autocuidado em saúde bucal ao paciente hospitalizado, a equipe disse recomendar o uso de escova dental, dentifrício e clorexidina $(68,4 \%)$ com frequência de três vezes ao dia (52,6\%).

Algumas perguntas resultaram em dados estatisticamente significativos, uma vez que se tornaram discordantes. Por exemplo, quando questionados: "A criança é encaminhada para atendimento odontológico?", $100 \%$ da equipe deenfermagem reportaram que sim, ao passo que apenas $23 \%$ dos cuidadores responderam o mesmo $(p=0,002)$. 0 mesmo pôde ser observado frente à pergunta "A criança relata desconforto na cavidade bucal?", em que $100 \%$ da equipe de enfermagem reportaram que sim, enquanto apenas $16 \%$ dos cuidadores responderam o mesmo que a equipe de enfermagem $(p<0,001)$.

Os desconfortos na cavidade bucal mais frequentemente reportados pela criança podem ser observados na Tabela 5. 
Tabela 1. Perfil das crianças hospitalizadas. HIJG, 2007.

\begin{tabular}{|c|c|}
\hline Característica & $\mathrm{n}$ \\
\hline \multicolumn{2}{|l|}{ Idade (anos) } \\
\hline 0 a 5 & 14 \\
\hline 6 a 10 & 14 \\
\hline Mais de 10 & 15 \\
\hline \multicolumn{2}{|l|}{ Sexo } \\
\hline Feminino & 12 \\
\hline Masculino & 31 \\
\hline \multicolumn{2}{|l|}{ Tempo de tratamento (meses) } \\
\hline Menos de 1 mês & 9 \\
\hline Até 6 meses & 7 \\
\hline 6 a 12 meses & 11 \\
\hline M ais de 12 meses & 3 \\
\hline Não responderam & 13 \\
\hline \multicolumn{2}{|l|}{ Tempo de internação (dias) } \\
\hline Até 7 & 28 \\
\hline 7 a 14 & 11 \\
\hline 14 a 21 & 1 \\
\hline Mais de 1 mês & 3 \\
\hline \multicolumn{2}{|l|}{ Diagnóstico (tipo de câncer) } \\
\hline Leucemia linfoblástica aguda & 21 \\
\hline Linfoma de Burkitt & 3 \\
\hline Neuroblastoma & 3 \\
\hline Osteossarcoma & 2 \\
\hline $\begin{array}{l}\text { Outros (estômago, rins, olhos, } \\
\text { intestino, pulmão, coração, } \\
\text { cérebro, abdômen, etc.) }\end{array}$ & 14 \\
\hline
\end{tabular}

Tabela 2. Perfil dos cuidadores (acompanhantes). HIJG, 2007.

\begin{tabular}{lrr}
\hline \multicolumn{1}{c}{ Característica } & $\mathrm{n}$ & $\%$ \\
\hline Idade (anos) & & \\
$\quad$ menos de 20 & 05 & 11,6 \\
21 a 30 & 10 & 23,3 \\
31 a 40 & 18 & 41,8 \\
mais de 40 & 10 & 23,3 \\
Sexo & & \\
Feminino & 36 & 83,7 \\
masculino & 07 & 16,3 \\
Grau de parentesco com a criança & & \\
$\quad$ Mãe & 34 & 79 \\
Pai & 07 & 16 \\
$\quad$ outros (tia, avó, etc.) & 02 & 5 \\
Estado civil & & \\
Casado (a) & 36 & 83 \\
$\quad$ Divorciado (a) & 04 & 10 \\
Solteiro (a) & 03 & 7 \\
Situação profissional & & \\
$\quad$ Empregado (a) & 16 & 41,8 \\
$\quad$ Desempregado (a) & 18 & 37,2 \\
Não trabalha & 08 & 14 \\
Aposentado (a) & 03 & 7 \\
& &
\end{tabular}

Tabela 3. Perfil da equipe de enfermagem. HIJG, 2007.

\begin{tabular}{lcr}
\hline \multicolumn{1}{c}{ Característica } & $\mathrm{n}$ & $\%$ \\
\hline Idade (anos) & & \\
20 a 30 & 02 & 10,6 \\
31 a 40 & 09 & 47,3 \\
M ais de 40 & 08 & 42,1 \\
Sexo & & \\
$\quad$ Feminino & 17 & 89,4 \\
$\quad$ masculino & 02 & 10,6 \\
Formação profissional & & \\
$\quad$ Curso técnico & 12 & 63,1 \\
$\quad$ Curso superior completo & 03 & 13,9 \\
$\quad$ Curso superior incompleto & 04 & 21 \\
\hline
\end{tabular}

\section{Discussão}

O Hospital Infantil Joana de Gusmão (HIJG), situado em Florianópolis, évinculado à Secretaria Estadual deSaúde(SES). É um hospital-escola, terciário, considerado polo dereferência estadual para as patologias de baixa, média e alta complexidade.

Atualmente, o setor de oncologia do HIJG possui catorze leitos, ea idade dos pacientes hospitalizados varia de alguns meses de vida até a pré-adolescência. Há também um ambulatório onde as crianças realizam tratamento antineoplásico e são encaminhadas para exames complementares. Este possui um cirurgião-dentista que realiza os atendimentos no consultório odontológico do ambulatório. Porém, a demanda é ainda maior, uma vez que, tanto as crianças hospitalizadas quanto as que fazem manutenção do tratamento antineoplásico recebem assistência odontológica.

Neste estudo, verificou-se um maior acome timento do câncer em crianças do sexo masculino (Tabela 1), o quetambém foi encontrado por $\mathrm{Hou}$ et al. ${ }^{17}$, Sepet et al. ${ }^{18}$, Silva et al. ${ }^{19}$ e Santos et al. ${ }^{20}$.

No Brasil, de cada dez a quinze casos de câncer, considerando-se a faixa etária abaixo de 15 anos, quatro são de leucemia linfoblástica agu$\mathrm{da}^{19,21,22}$, o que demonstra que a leucemia é 0 diagnóstico mais frequenteem crian ças ${ }^{10,20,23}$, fato este também observado nos dados obtidos neste estudo, cuja prevalência foi de $48 \%$. 
Tabela 4. Conhecimentos e práticas de higiene bucal. HIJG, 2007.

\begin{tabular}{|c|c|c|c|c|}
\hline \multirow{2}{*}{ Conhecimentos e práticas } & \multicolumn{2}{|c|}{ Cuidadores } & \multicolumn{2}{|c|}{ Enfermagem } \\
\hline & $\mathrm{n}$ & $\%$ & $\mathrm{n}$ & $\%$ \\
\hline \multicolumn{5}{|l|}{ Vocês receberam instruções de HB? } \\
\hline Sim & 28 & 65,11 & 08 & 42,1 \\
\hline Não & 15 & 34,89 & 11 & 57,9 \\
\hline \multicolumn{5}{|l|}{ Quem forneceu as informações sobre HB? } \\
\hline Equipe médica & 12 & 42,8 & - & - \\
\hline Equipe de enfermagem & 06 & 21,4 & - & - \\
\hline Cirurgião-dentista & 08 & 28,5 & - & - \\
\hline Cursos, palestras & - & - & 08 & 100 \\
\hline Leitura & 02 & 7,3 & - & - \\
\hline \multicolumn{5}{|l|}{ Você realiza a H B da(s) criança (s)? } \\
\hline Sim & 39 & 90,7 & - & - \\
\hline Não & 04 & 9,3 & 18 & 94,7 \\
\hline às vezes & - & - & 01 & 5,3 \\
\hline \multicolumn{5}{|l|}{ Com que freqüência é realizada a HB? } \\
\hline 2 vezes ao dia & 12 & 28 & - & - \\
\hline 3 vezes ao dia & 17 & 39,5 & - & - \\
\hline M ais de 3 vezes ao dia & 14 & 32,5 & - & - \\
\hline \multicolumn{5}{|l|}{ Com o que realiza a $\mathrm{HB}$ ?/ O que indica para a $\mathrm{HB}$ ? } \\
\hline Escova, dentifrício & 07 & 16,2 & 01 & 5,3 \\
\hline Escova, dentifrício e fio dental & 01 & 2,4 & - & - \\
\hline Escova, dentifrício e clorexidina & 27 & 62,8 & 13 & 68,4 \\
\hline Escova, dentifrício, fio dental e clorexidina & 05 & 11,6 & 01 & 5,3 \\
\hline Clorexidina & 02 & 4,6 & 04 & 21 \\
\hline Bicarbonato de sódio & 01 & 2,4 & - & - \\
\hline \multicolumn{5}{|l|}{ A criança relata desconforto na cavidade bucal? } \\
\hline Sim & 16 & 62,8 & 19 & 100 \\
\hline Não & 27 & 37,2 & - & - \\
\hline \multicolumn{5}{|c|}{ A criança é encaminhada para o cirurgião-dentista? } \\
\hline Sim & 23 & 53,4 & 19 & 100 \\
\hline Não & 20 & 46,6 & - & - \\
\hline \multicolumn{5}{|c|}{ Você acha importante ter um cirurgião-dentista na equipe? } \\
\hline Sim & 43 & 100 & 19 & 100 \\
\hline Não & - & - & - & - \\
\hline
\end{tabular}

Tabela 5. Prevalência das manifestações clínicas relatadas pelos cuidadores e equipe de enfermagem durante a terapêutica antineoplásica. HIJG, 2007.

\begin{tabular}{|c|c|c|c|c|}
\hline \multirow{2}{*}{ M anifestações clínicas } & \multicolumn{2}{|c|}{ Cuidadores } & \multicolumn{2}{|c|}{ Enfermagem } \\
\hline & $n$ & $\%$ & $n$ & $\%$ \\
\hline Dor na mucosa & 12 & 75 & 16 & 84,2 \\
\hline Enjôos & 09 & 56,25 & 14 & 73,6 \\
\hline Vômitos & 09 & 56,25 & 14 & 84,2 \\
\hline Ardência & 03 & 18,75 & 16 & 84,2 \\
\hline Boca seca & 04 & 25 & 05 & 26,3 \\
\hline Dor de dente & 03 & 18,75 & 03 & 15,7 \\
\hline Ausência de paladar & 01 & 6,25 & 01 & 5,2 \\
\hline
\end{tabular}


Analisando-se a faixa etária de ocorrência do câncer, observou-se que na amostra estudada houve distribuição si milar entre as idades de 0-5 anos (32,5\%) e 6- 10 anos (32,5\%), sendo observado o mesmo no estudo de Santos et al. ${ }^{20}$, cuja prevalência foi de $68,57 \%$ em crianças com até dez anos de idade.

Dentre as terapêuticas para o tratamento do câncer estão a quimioterapia, radioterapia, cirurgia, ou a associação delas ${ }^{10,24}$. 0 tratamento mais utilizado para as leucemias é a quimioterapia intensiva em três fases: indução, consolidação e manutenção 25 .

Os principais efeitos colaterais da terapêutica antineoplásica são a mucosite, a xerostomia temporária e a imunodepressão, possibilitando infecções dentárias ou oportunistas. O bservam-se também hemorragias gengivais decorrentes da plaquetopenia e distúrbios na formação dos germes dentários, quando a quimioterapia é administrada na fase de odontogênese $\mathrm{e}^{14 \cdot 16}$.

Dentre os desconfortos mais frequentes neste estudo (Tabela 5), constatou-se a "dor na mucosa" (mucosite), em virtude da presença de ulcerações. Santos et al. ${ }^{20}$ observaram que durante a terapia, $81,82 \%$ tiveram a mucosa bucal comprometida por ulcerações, sangramento gengival ecandidíase. Ribas e Araújo ${ }^{23}$, no entanto, observaram uma menor prevalência de manifestações bucais nosportadores deleucemia na infância; $17,64 \%$ de lesões ulceradas e $11,76 \%$ com gengivite.

A mucositeéuma inflamação da mucosa, frequente edolorosa, queocorre de cinco a sete dias após a terapia antineoplásica (quimio/radio) e que depende do grau de perda tecidual e agressão de patógenos. Essa alteração na mucosa pode progredir para a descamação celular, resultando em úlceras sintomáticas, dificultando a fala e a alimentação ${ }^{20} .0$ aparecimento delesões na cavidade bucal em relação ao período inicial do tratamento oncológico comprova os dados achados nesta pesquisa (Tabela 1 e Tabela 5).

Muitos dos agentes quimioterápicos são administrados por via sistêmica e provocam distúrbios horas depois de serem administrados, aumentando o risco dos efeitos agressi vos à saúde bucal no curso do tratamento ${ }^{20}$. As sintomatologias como enjoos e vômitos foram relatados com grande frequência (Tabela 5).

A sensação de boca seca (xerostomia) também foi um dos desconfortos presentes nos resultados desta pesquisa, e o padrão de resposta foi similar entre os pesquisados (Tabela 5). Apesar de ser considerado um dos efeitos mais frequentes da irradiação de cabeça e pescoço ${ }^{24}$, nessa po- pulação estudada a prevalência foi menor do que a apresentada por outros estudos na literatura.

Como consequência da xerostomia ocorre uma hipofunção das glândulas salivares, que consequentemente, acarreta em alterações na dieta, que por sua vez alteram a microflora (produzem um aumento de Streptococcus mutans), e essa alteração, somada à precária higiene bucal observada, favorece o surgimento de lesões de cárie $^{24-27}$. Outra explicação para 0 aumento na incidência de lesões de cárie pode ser a ausência de paladar (disgeusia), o que sugere a mudança na alimentação do paciente para uma dieta mais macia edoce.

A familiarização com todos os possíveis sintomas associados aos pacientes oncológicose uma subsequente generalização das sequel as do tratamento antineoplásico por parte da equipe de enfermagem, de um lado, e o fato de os cuidadores usarem suas experiências pessoais, por outro lado - ambos quando questionados -, pode ser a causa da discordância de respostas com relação ao desconforto na cavidade bucal $(p<0,001)$.

Pelo fato de os pacientes serem crianças, suas mães estavam presentes durante as consultas e, consequentemente, durantea realização do estudo (Tabela 2). Assim, observou-se que a presença delas indica o importante papel que possuem dentro do núcleo familiar. Além disso, acreditase que a presença das mães tenha diminuído a ansi edade das crianças frente ao tratamento em âmbito hospitalar ${ }^{28}$.

Sabe-se, na prática da odontologia, que os hábitos adquiridos pelas crianças para a promoção de saúde bucal se estabelecem através da observação das práticas de prevenção da mãe. As crianças cujas mães têm pouco cuidado apresentam forte tendência a também desenvolver poucos cuidados ${ }^{29}$, tornando-se um ponto chave no processo de prevenção ${ }^{30}$.

Altamirano e Jereissati ${ }^{31}$ destacaram que as orientações desaúde bucal, por mais simples que sejam, oferecem resultados positivos e são essenciais durante o período de internação de crianças em enfermarias pediátricas dehospitais públicos. Em tais enfermarias, o perfil da população atendida écarente, o número ea rotatividade de pacientes são elevados e não há condições de consultas frequentes serem realizadas.

Quando os cuidadores foram questionados sobre conhecimentos e práticas em saúde bucal (Tabela 4), observou-se que a grande maioria considerava-se informada e que realizavam a higienização bucal de seus filhos por meio de escova, dentifrício e clorexidina. 
A explicação para o uso da clorexidina foi encontrada quando a equipe de enfermagem informou não haver um protocolo de higienização bucal, mas que recomendavam o uso diário de escova dental edentifrício e o bochecho com clorexidina (Tabela 4).

Poucos estudos na literatura são referentes à avaliação de um protocolo que seja efetivo para inibir ou amenizar as sequelas bucais do tratamento contra o câncer. Costa et al. ${ }^{32}$ anal isaram a efetividade de um protocolo preventivo utilizando a clorexidina na prevenção das complicações bucais durante 0 tratamento de quimioterapia para a LLA. Os autores observaram nas crianças que fizeram uso do protocolo preventivo um menor desenvolvimento de complicações bucais, apesar de reportarem sensação de ardência.

É importante ressaltar que apesar de ser considerado um antimicrobiano altamente eficaz, o uso da clorexidina deve ser recomendado apenas nos casos em que o paciente não consegue realizar a higienebucal. Como qualquer outro agente antimicrobiano potente, deve ser administrada somentesob supervisão profissional epossui efeitos colaterais quando utilizada por longos períodos. Exemplos desses efeitos colaterais são: pigmentação dos dentes, descamação do dorso da língua, sensibilidade oral (ardência) e alteração da gustação após algumas horas ${ }^{33}$.

M ediante tal afirmação, os participantes do estudo foram questionados sobre a importância da participação efetiva de um cirurgião-dentista, em ambiente hospitalar, para que medidas de promoção de saúde fossem tratadas com prioridade. As repostas demonstraram que os participantes, de forma unânime (Tabela 4), acreditam que seria muito importante a inserção do $C D$ neste ambiente.

Outra divergência de respostas foi referente ao questionamento "A criança é encaminhada para atendimento odontológico?" ( $p=0,002)$. Segundo os cuidadores, muitos deles não sabiam da existência de um cirurgião-dentista na equipe médica. Tal fato sugere a necessidade de maiores esclarecimentos à população, quer seja durantea entrada no HIJG ou por folhetos informativos distribuídos no setor de oncologia. Caso essas medidas tivessem sido adotadas, muitas seque las poderiam ter sido minimizadas e uma me Ihora da qualidade de vida promovida.

Constatou-se, também, que os profissionais da saúdecom maior contato erelação direta com os pacientes durante o período deinternação são provenientes da equipedeenfermagem, composta por enfermeiros etécnicos em enfermagem. Sen- do assim, os mesmos foram questionados quanto aos conhecimentos sobre rotina dehigienebucal. D entreosdozetécnicos em enfermagem, apenas três reportaram ter recebido orientações, enquanto cinco das sete enfermei ras reportaram 0 mesmo (Tabela 4).

Essa informação sugere a existência de uma dissociação do que se entende por higiene, assim como por saúde. Talvez isso se deva a questões defalta deconhecimentos específicos, insegurança, receio, sentimento de inaptidão para exercer tal função ou, até mesmo, pela ausência do pensar na integralização do paciente.

Essa ausência do pensar na saúde bucal como parte da higiene geral torna-se evidente ao analisarmos as atribuições dos técnicos em enfermagem, especificadas pelo código de ética da entida$\mathrm{de}^{34}$ (Art. 11, item IV). Segundo tal código, éconsiderado dever dos mesmos "[...] prestar cuidados de higiene [...]". Deacordo com essa afirmação, ser responsável pela higienedo paciente não se traduz em ser responsável, também, pela higiene bucal. Esse fato pôde ser comprovado neste estudo (Tabela 4), uma vez que apenas um funcionário reportou realizar, às vezes, a higiene bucal do paciente infantil hospitalizado.

Porém, esse fato de não se considerar o paciente como um todo pode ser observada em todos os segmentos da área da saúde e não somente na enfermagem. Esse pensamento coletivo errôneo resulta em prejuízo no restabelecimento integral do paciente e na qualidade de vida do mesmo edemonstra um déficit na formação profissional do técnico em enfermagem.

De acordo com Cecílio e Merhy ${ }^{35}$, o cuidado com o paciente hospitalizado depende da interação do trabalho multiprofissional, resultado da soma de pequenos cuidados parciais que se complementam. Existe, no entanto, uma dificuldade em seestabelecer funções e delegar responsabilidades, o que resulta em uma sobrecarga no processo de gerência de um hospital. Torna-se um desafio coordenar adequadamente uma equipe tão diversificada e especializada de profissionais da saúde.

Nesse contexto hospitalar, talvez seja benéfico para um efetivo desenvolvimento das atividades rotineiras a presença de um CD, cuja responsabilidade seja motivar econscientizar osfuncionários sobre a importância dos cuidados bucais para o restabelecimento da saúde, já que atuam como um ativador demudanças. E, queesse profissional oportunize o vínculo entre a equipe de enfermagem eo paciente hospitalizado para que os objetivos em tornar a saúde integralizada sejam consolidados. 
Assim, é necessário que se inicie um movimento de educação para a saúde e que os profissionais se organizem de forma não necessariamente hierárquica, mas sim em uma divisão de atribuições baseada em suas habilidades, que quando agregadas promovam a transformação das atitudes, das representações sociais e dos comportamentos ${ }^{36}$.

Espera-se, dessa forma, que com a motivação na transformação das atitudes, os responsáveis pelas crianças entendam que inúmeras sequelas advindas do tratamento antineoplásico podem ser evitadas ou amenizadas e que 0 acometimento da cavidadebucal mediantetal intervenção médica não é uma obrigatoriedade ou uma fatalidade.

0 desfecho desse movimento é difícil e demorado, mas, se ao ser iniciado, houver o entendimento dos limites e das dificuldades do processo educativo, como e a quem educar, flexibilidade para adequação e adaptação dos modelos propostos inicial mente, haverá uma grande possibilidade de se alcançar os objetivos propostos para uma educação em saúde ${ }^{36}$.

\section{Colaboradores}

AM Barbosa trabalhou em todas as etapas do projeto e elaboração final do trabalho. DM Ribeiro participou da concepção e redação final. AS Caldo-Teixeira participou da análise dos resultados e redação final.

\section{Agradecimentos}

Ao diretor geral do HIJG, Dr. Maurício Laerte Silva, ao chefe do setor de oncologia, Dr. Lincoln Virmond Abreu, e ao coordenador da odontologia do HIJG, Dr. Levy Hermes Rau, por possibilitarem a realização deste estudo. À equipe de enfermagem e às crianças e suas famílias por partici parem deforma tão solícita durantea execução do estudo. À Laura Scarparo Caldo Teixeira pela revisão gramatical do texto.

\section{Consideraçõesfinais}

De acordo com os resultados obtidos, pôde-se concluir que não existe um protocolo de cuidados com a higiene bucal de crianças hospitalizadas com câncer e que as manifestações bucais mais frequentes entre os pacientes em tratamento antineoplásico foram: mucosite, enjoos, vômitos, xerostomia e ausência de paladar.

Dessa forma, é de fundamental importância que se estabeleça um protocolo de cuidados com a higiene bucal de crianças hospitalizadas com câncer, uma vez que as mesmas encontram-se vulneráveis nesse período. A presença de um cirurgião-dentista na equipe médica parece reforçar a preocupação em minimizar os danos provenientes do tratamento oncológico e pode, a partir de suas atribuições e habilidades, ser um agente ativador de mudanças em educação para a saúde.

Há a necessidade de um tratamento profilático que amenize as manifestações bucais em decorrência do tratamento oncológico, visando uma melhoria na qualidade de vida do paciente hospitalizado. 


\section{Referências}

1. Ferreira MCM, Orsi RM. A saúde bucal ao alcance de todos. São Paulo: Santos; 1995.

2. Abegg $\mathrm{C}$. Hábitos de higiene bucal de adultos portoalegrenses. Rev. Saúde Publica 1997; 31(6):586-593.

3. Martins ALCF, Fernandes FRC, Corrêa M SNP, Guerrera $A C$. A cárie dentária. In: Corrêa M SN P, organizador. 0 dontopediatria na primeira infância. $1^{\underline{a}} \mathrm{ed}$. São Paulo: Santos; 1998.

4. Costa CC, Costa Filho LC, Sória ML, M ainardi APR. Plaque removal by manual and eletric toothbrushing among children. Pesqui. Odontol Bras 2001; 15(4):269-301.

5. Honkala E. Oral health promotion withchildren and adolescents. In: Schou L, Blinkhorn A, editors. Oral health promotion. Oxford: Oxford University; 1993.

6. Araújo $A$. Estudo das condições de saúde bucal e necessidades detratamento em pacientes do curso de odontologia da Universidade Federal do Pará [dissertação]. São Paulo (SP): Universidade Federal de São Paulo; 2003.

7. Guedes-Pinto AC. Odontopediatria. 6a ed. São PauIo: Santos; 2000.

8. Lascala NT. Prevenção na clínica odontológica: promoção de saúde bucal. São Paulo: Artes M édicas; 1997.

9. Goursand D, Borges CM, Alves KM, Nascimento $A M$, Winter RR. Sequelas bucais em crianças submetidas à terapia antineoplásica: causas e definição do papel do cirurgião dentista. Arquivos em Odontologia 2006; 42(3):161-256.

10. Braga PE, Latorre MRDO, Curado M P. Câncer na infância: análise comparativa da incidência, mortalidade e sobrevida em Goiânia (Brasil) e outros países. Cad Saude Publica 2002; 18(1):33-44.

11. Brasil. M inistério da Saúde. Incidência de câncer no Brasil. [site da Internet]. 2006. Disponível em: http:/ /www.inca.gov.br

12. Precioso VC, Esteves ARF, Souza AM, Dib LL. Complicações orais na quimioterapia em oncologia pediátrica: o papel da odontologia preventiva. Acta Oncol Bras 1994; 14:147-152.

13. Sonis ST, Fazio RC, Fang L. Princípios e prática de medicina oral. $2^{a}$ ed. Rio de Janeiro: Guanabara Koogan; 1996.

14. Rey E, Michelet MC. Tratamiento de las complicaciones bucales en pacientes oncohematológicos, durante la granulocitopenia producida por la quimioterapia. Rev Fac Odontol 1994; 14(35):70-76.

15. Minicucci EM, Dib LL, Curi MM, Shinohara EH, Sêneda LM. Sequelas odontológicas do tratamento rádio e quimioterápico em crianças. Rev Paul Pediatr 1994; 12:258-263.

16. Fonseca MA. Pediatric bone marrow transplantation: oral complications and recommendations for care. Pediatr Dent 1998; 20(7):386-394.

17. Hou GL, Huang JS, Tasi CC. Analysis of oral manifestations of leukemias: a retrospective study. Oral Dis 1997; 3(1):31-38.
18. Sepet E, Aytepe Z, Ozerkan AG, Yalman N, Guven Y, Anak S, Devecioglu O, Agaoglu L, Gedikoglu G. Acute lymphoblastic leukemia: dental health of children in maintenance therapy. J Clin Pediatr Dent 1998; 22(3):257-260.

19. Silva DB, Pires MMS, Nassar SM. Câncer pediátrico: análise de um registro hospitalar. J. Pediatr (Rio J) $2002 ; 78(5): 409-414$.

20. Santos VI, Anbinder AL, Cavalcante ASR. Leucemia no paciente pediátrico: atuação odontológica. Cienc Odontol Bras 2003; 6:49-57.

21. Ribas MO, Costa NP. Estudo das observações clínicas sistêmicas, estomatológicas e radiográficas das alterações dentárias e ósseas nos pacientes com leucemia na infância. Rev Odontol Cienc 1995; 10(20):151-184.

22. Orbak R, Orbak Z. Oral condition of patients with leukemia and lymphoma. J Nihon Sch Dent 1997; 39(2):67-70.

23. Ribas M O, Araújo M R. Manifestações estomatológicas em pacientes portadores de leucemia. Rev. Clín. Pesq. Odontol. 2004; 1(1):35-41.

24. Cardoso MFA, Novikoff S, Tresso A, Segreto RA, Cervantes 0 . Prevenção e controle das sequelas bucais em pacientes irradiados por tumores de cabeça e pescoço. Radiol Bras 2005; 38(2):107-115.

25. Kroetz FM, Czlusniak GD. Alterações bucais e condutas terapêuticas em pacientes infanto-juvenis submetidos a tratamentos anti-neoplásicos. Publ. UEPG Biol. Health Sci 2003; 9(2):41-48.

26. Jensen SB, Pedersen AM, Reibel J, Nauntofte B. Xerostomia and hypofunction of the salivary glands in cancer therapy. Support Care Cancer 2003; 11:207-225.

27. Camargo JDF, Batistella FID, Ferreira SLM. Complicações bucais imediatas do tratamento oncológico infantil: identificação, prevenção e tratamento. Rev Ibero-am O dontopediatr O dontol Bebê 2004; 7(36):177-184.

28. Virdee PK, Rodd HD. Who accompanies children to a dental hospital appointment? European Archives of Paediatric Dentistry 2007; 8(2):95-98.

29. Zardetto CGC, Rodrigues CRM D, Ando T. Avaliação dos conhecimentos de alguns tópicos de saúde bucal de gestantes de níveis sócio-culturais diferentes. RPG Rev Pós Grad 1998; 5(1):69-74.

30. Costa ICC. A gestante como agente multiplicador de saúde. RPG Rev Pós Grad 1998; 5(2):87-92.

31. Altamirano EHD, Jereissati L. A fisioterapia respiratória e o processo de hospitalização criança-mãe. Psicologia: teoria e prática 2002; 4(2):57-65.

32. Costa EM M B, Fernandes M Z, Quinderé LB, Souza LB, Pinto LP. Evaluation of an oral preventive protocol in children with acute lymphoblastic leukemia. Pesqui Odontol Bras 2003; 17(2):147-150.

33. Egbert $B$, Toledo $C$, Junior $C R$. Influências das condições sistêmicas sobre as doenças periodontais e das doenças periodontais sobre as condições sistêmicas. In: Tunes UR, Rapp GE, organizadores. Atualização em periodontia e implantodontia. São Paulo: Artes M édicas; 1999. p. 31-55. 
34. Conselho Regional de Enfermagem de Santa Catarina. COREN-SC ao seu alcance. 6a ed. Florianópolis: COREN-SC; 2004.

35. Cecilio LCO, M erhy EE. Integralidade do cuidado como eixo da gestão hospitalar. In: Pinheiro R, $M$ attos $R$, organizadores. Construção da integralidade: cotidiano, saberes e práticas em saúde. Rio de Janeiro: IM S/ABRASCO; 2003.

36. Mello MS, Ferreira EF, Paixão HH. Educação para saúde em hospital - relato de uma experiência. Arq Centro Est Curso Odont 1992; 29(2):99-103.

Artigo apresentado em 30/11/2007

Aprovado em 21/07/2008

Versão final apresentada em 15/08/2008 